\title{
Erratum to: Bias Related to Body Mass Index in Pediatric Echocardiographic $Z$ Scores
}

\author{
Frederic Dallaire $^{1,2} \cdot$ Jean-Luc Bigras ${ }^{3} \cdot$ Milan Prsa $^{4} \cdot$ Nagib Dahdah $^{3}$
}

Published online: 14 June 2015

(c) Springer Science+Business Media New York 2015

\section{Erratum to: Pediatr Cardiol (2015) 36:667-676 \\ DOI 10.1007/s00246-014-1063-7}

The original version of this article unfortunately contained an error in the supplementary material of the original publication.

The presentation of Table 3 was incorrect in the supplementary material. The corrected version of the supplementary material is uploaded with this erratum, which can be accessed using the DOI given below.

The online version of the original article can be found under doi:10.1007/s00246-014-1063-7.

Electronic supplementary material The online version of this article (doi:10.1007/s00246-015-1212-7) contains supplementary material, which is available to authorized users.

Frederic Dallaire

frederic.a.dallaire@usherbrooke.ca

1 Division of Pediatric Cardiology, Department of Pediatrics, Faculty of Medicine, University of Sherbrooke, 3001, 12e Avenue Nord, Sherbrooke, QC J1H 5N4, Canada

2 Centre de Recherche du Centre Hospitalier Universitaire de Sherbrooke (CHUS), Sherbrooke, QC, Canada

3 Division of Pediatric Cardiology, Sainte-Justine University Hospital Center, University of Montreal, Montreal, QC, Canada

4 Division of Pediatric Cardiology, Department of Pediatrics, University Hospital of Lausanne, Lausanne, Switzerland 